\title{
Use of genomic panels to determine risk of developing type 2 diabetes in the general population: a targeted evidence-based review
}

\author{
Glenn E. Palomaki, $\mathrm{PhD}^{1}$, Stephanie Melillo, $\mathrm{MPH}^{2,3}$, Michael Marrone, $\mathrm{MPH}^{2,4,5}$ and \\ Michael P. Douglas, MS',4
}

\begin{abstract}
This evidence review addresses whether type 2 diabetes genomic risk panels improve health outcomes (e.g., reduce rates of developing type 2 diabetes) in low- or high-risk adults; two clinical scenarios promulgated by commercial companies offering such testing. Evidence for the analytic validity of available genomic profiles was inadequate. Clinical validity ranged from inadequate to convincing for 30 variants identified on five type 2 diabetes genomic panels and by genomewide association studies. Eight common variants were identified for general population use; evidence credibility based on published criteria was strong for two variants, moderate for two variants, and weak for four variants. TCF7L2 had the largest per-allele odds ratio of 1.39 (95\% confidence interval 1.33-1.46). Models combining the best four, best eight, and all 30 variants used summary effect sizes, reported genotype frequencies, and assumed independent effects.
\end{abstract}

\section{INTRODUCTION}

Diabetes mellitus refers to a group of metabolic diseases that are characterized by chronic elevations in plasma glucose. Up to $95 \%$ of all diabetes is considered type 2 diabetes (T2D), which was previously known as "adult-onset" or "noninsulin-dependent diabetes." The metabolic mechanisms for T2D include insulin resistance, impaired insulin secretion, and increased hepatic glucose production. Complications can include nephropathy, neuropathy, retinopathy, periodontal disease, and accelerated development of cardiovascular disease. Approximately 1.9 million people aged 20 years or older were newly diagnosed with diabetes in 2010 in the United States. ${ }^{1}$ T2D is becoming more common in the United States and throughout many regions of the world. The T2D prevalence in youths aged 10 years and older is 8.5 per 100,000, whereas the prevalence is 0.4 per 100,000 in youths younger than 10 years. ${ }^{1}$ The prevalence is even higher among adults aged 65 years and older, with 10.9 million, or $26.9 \%$, of all people in this age group being affected. ${ }^{1}$ However, the Centers for Disease Control and Prevention estimates as many as $27 \%$ of individuals with T2D are undiagnosed. Factors such as an aging population and rising rates of obesity are expected to further increase the prevalence.

The prevalence of T2D varies by race and ethnicity, with higher rates among African Americans (11.4\%), Hispanic/
Areas under the curve were $0.547,0.551$, and 0.570 , respectively. In high-risk populations, per-allele odds ratios for TCF7L2 alone were similar to those of the general population. TCF7L2, in combination with other variants, yielded minimal improvement in risk reclassification. Evidence on TCF7L2 clinical validity was adequate. Three studies addressed the clinical utility of intervention effectiveness, stratified by TCF7L2 genotype; none found significant interactions. Clinical utility evidence was inadequate. In addition to analytic validity and clinical utility knowledge gaps, additional gaps were identified regarding how to inform, produce, and evaluate models combining multiple variants. Genet Med 2013:15(8):600-611

Key Words: clinical utility; clinical validity; evidence-based review; genomic panels; type 2 diabetes

Latino Americans (8.2\%), Native Americans (8.2\% among Alaska Natives, $27.8 \%$ among certain Native American tribes from the Southwest, and up to 50\% among Pima Indians and some Asian-American and Pacific Islander groups). Randomized trials have demonstrated that changing lifestyle factors (e.g., weight loss, improved diet, and increased physical activity) can help prevent or delay the development of diabetes. ${ }^{2-9}$

A family history of diabetes is a major risk factor for the disease and is often included in tools designed to quantify the risk of diabetes, ${ }^{10}$ with odds ratios (ORs) of two to six, if one or more first-degree relatives have been diagnosed with T2D. ${ }^{11}$ A long-term study reported that the cumulative prevalence of T2D at 80 years of age is approximately 3.5 times higher $(38 \%$ vs. $11 \%$ ) for individuals with a first-degree relative diagnosed with T2D as compared with individuals without any relatives diagnosed with T2D. ${ }^{12}$

Several commercial and noncommercial entities are now offering diabetes risk testing alone or as a part of a panel involving risk profiling for chronic diseases. If these gene panels were to demonstrate the capacity of identify a subgroup with important increases in the risk of developing diabetes, it might be possible to implement targeted prevention strategies that might be too invasive or too resource intensive to be offered to

${ }^{1}$ Department of Pathology and Laboratory Medicine, Women \& Infants Hospital, Alpert Medical School of Brown University, Providence, Rhode Island, USA; ${ }^{2}$ Office of Public Health Genomics, Centers for Disease Control and Prevention, Atlanta, Georgia, USA; ${ }^{3}$ Division of Cancer Prevention and Control, Centers for Disease Control and Prevention, Atlanta, Georgia, USA; ${ }^{4}$ McKing Consulting Corporation, Atlanta, Georgia, USA; ${ }^{5}$ Johns Hopkins Bloomberg School of Public Health, Baltimore, Maryland, USA. Correspondence: Glenn E. Palomaki (gpalomaki@ipmms.org) 
a general population. Such testing and targeted interventions have the potential to reduce the burden of disease.

The Evaluation of Genomic Applications in Practice and Prevention Working Group (EWG) was established in 2005 to support the development of a systematic process for assessing the available evidence for rapidly emerging genetic tests for clinical practice. This independent, multidisciplinary panel selected this topic to commission an evidence report to review validity and utility and highlight critical knowledge gaps, in order to provide guidance on appropriate use of the available genetic tests in specific clinical scenarios. T2D risk panels were selected for evaluation by Genomic Applications in Practice and Prevention because of the prevalence and burden of T2D. Testing is offered through both clinical and direct-to-consumer models, each with unique considerations, and for two clinical scenarios. Estimating the risk of T2D on the basis of variants in multiple genes, individually and in combination, challenges risk assessment and Genomic Applications in Practice and Prevention evidence review methods. The analytic validity (technical test performance), clinical validity (the strength of association that determines the test's ability to accurately and reliably identify or predict the disorder of interest), and clinical utility (balance of benefits and harms when the test is used to influence patient management) are systematically reviewed. ${ }^{13}$

The EWG chose to examine test performance in two separate clinical scenarios that have been promulgated by those offering clinical testing. The first was a claim that a gene panel consisting of multiple markers could identify adults from the general population who would be at high risk of developing T2D. These individuals could then be offered targeted interventions to reduce the likelihood of developing T2D in the future. The second scenario involved testing adults who had already been identified as being at high risk of developing T2D in the near future. At the time the review was commissioned, claims for this scenario involve testing for only one gene (TCF7L2). The aim of the review is not to provide a comprehensive review of all potential genomic markers for T2D. According to Phenopedia (www.cdc.gov/genomics/hugenet/hugenavigator.htm), there are 3,267 genes already studied in relation to T2D, with 208 meta-analyses and 106 genome-wide association (GWA) studies, with several hundred publications added per year. Rather, this review focuses on what T2D panels are clinically available and determine through an overview of additional markers whether it is reasonable to assume that important advances in the clinical validity of these panels are expected.

\section{METHODS}

\section{Overview}

The targeted evidence-based review process used to identify, review, analyze, evaluate, and summarize the evidence is briefly presented here, but an in-depth presentation of EWG methods has been published..$^{13} \mathrm{~A}$ consultant from Women \& Infants Hospital's Department of Pathology and Laboratory Medicine (G.E.P.) with experience in evidence review of genetic tests was contracted by the Office of Public Health Genomics at the
Centers for Disease Control and Prevention to assist in the targeted review by the three members of that office (M.P.D., S.M., and M.M.). Guidance was provided by a technical expert panel, whose members are listed in the Acknowledgments section.

An analytic framework and key questions for two clinical scenarios (Table 1, Figure 1) were developed by the EWG with support from Genomic Applications in Practice and Prevention staff and refined through discussion at technical expert panel calls and EWG meetings. The review not only focuses on clinical validity but also addresses the limited information available on the platforms used for testing (analytic validity) and potential evidence for clinical utility both in clinical practice and in direct-to-consumer settings.

Standard methods were used, including systematic searches of published literature, limited identification of gray literature, use of inclusion/exclusion criteria, abstraction of data, metaanalysis, assessment of individual study quality, and grading of overall strength of evidence. ${ }^{13}$ Details on these methods as applied to this study are provided in the relevant sections below. In reviewing the available evidence, questions from the ACCE (Analytic validity, Clinical validity, Clinical utility, and Ethical, legal and social implications) review framework were often used to identify and organize information. ${ }^{14}$

\section{Analytic validity}

Targeted PubMed ${ }^{15}$ searches were performed for the alleles/ single-nucleotide polymorphisms (SNPs) and specific terms with the expectation of identifying published papers reporting on the analytic validity of methods specific to these genomic panels. For example, the PubMed search for TCF7L2 was as follows: "TCF7L2 OR TCF7L2 genotyping" AND "analytic validity OR clinical test." The same search was performed for each of the variants listed in Table 2. Gray literature searches (e.g., company and genetic testing websites) were also conducted to collect any available information on laboratories offering testing for these markers and the methodologies used.

\section{Clinical validity}

To increase efficiency, the review process was limited in the following ways: (i) inclusion of only published studies that reported T2D as the primary outcome, (ii) restriction of examined genes/polymorphisms to those included in T2D panels available in the United States in August 2010, and (iii) use of existing meta-analyses, when possible. Existing meta-analyses were considered acceptable if they were published in the past several years, reported summary effect size, included a formal analysis of heterogeneity, and examined the potential for publication bias. Searches of the published literature were conducted using HuGE Navigator v2.0. ${ }^{16,17}$ We have shown in a previous targeted review that this is a more specific search strategy that maintains sensitivity for identifying appropriate articles for review equivalent to PubMed. ${ }^{18}$ Specific search strategies for each gene are contained in Supplementary Appendix A online. Reference lists of retrieved publications were also examined to identify relevant studies. Searches began in August 2010 and 
Table 1 Key questions related to the analytic framework for both clinical scenarios

Clinical scenario 1: general population

1. Does the use of the T2D Multigene Panel test(s) lead to improved outcomes for the patient/consumer, or is it useful in medical or personal decision making? (overarching question)

2. What is known about the analytic validity of the test(s) used to identify the variations in the genes in the T2D Multigene Panel, including the analytic sensitivity and specificity, reproducibility, assay robustness (e.g., failure rates, resistance to changes in variables such as sample quality), and other factors?

3. What is the clinical validity of the T2D Multigene Panel, including clinical sensitivity and specificity and positive and negative predictive values?

a. What is the strength of association of the variants in these specific genes with risk for T2D (e.g., odds ratios)?

b. How well does this testing alone predict risk for T2D?

c. How well does this testing in combination with other clinical factors (e.g., family history, age, BMI/obesity, and glucose levels) predict risk for T2D?

d. How do other genetic and environmental factors (e.g., race/ethnicity, family history, smoking, diet, exercise level, and other conditions) affect the clinical validity of this test?

4. What are the issues related to the use of the T2D Multigene Panel test in the general population and its impact on patient/consumer outcomes?

a. What are the current management options for patients/consumers at risk for T2D based on a positive T2D Multigene Panel result in a medical model? How would recommendations differ from routine health messages? How would outcomes change based on use of this test in a direct-toconsumer model?

b. How could the results of the T2D Multigene Panel for risk of T2D in the general population impact health behaviors or inform decision making by patients and their health-care providers that affect outcomes?

c. In what ways could the use of the T2D Multigene Panel in the general population impact clinical outcomes (e.g., morbidity/mortality)?

d. What is known about other contextual issues, such as cost-effectiveness, likelihood of behavioral change, and family history considerations?

5. What are the potential harms associated with use of the T2D Multigene Panel (e.g., marketing direct to consumers, distress or stigma for a "poor prognosis" result, misinterpretation of results leading to excessive or inadequate treatment, exploitation of hyper-vigilant people)?

Clinical scenario 2: high-risk population

1. Does the use of TCF7L2 testing lead to improved outcomes for the high-risk ${ }^{\text {a }}$ patient/consumer, or is it useful in medical or personal decision making? (overarching question)

2. What is known about the analytic validity of the test(s) used to identify the variation in the TCF7L2 gene, including the analytic sensitivity and specificity, reproducibility, assay robustness (e.g., failure rates, resistance to changes in variables such as sample quality), and other factors?

3. What is the clinical validity of TCF $L 2$ testing, including clinical sensitivity and specificity and positive and negative predictive values?

e. What is the strength of association of the variant in this specific gene with short-term risk for T2D (e.g., odds ratios)?

f. How well does this testing alone predict risk for T2D?

g. How well does this testing in combination with other clinical factors (e.g., family history, age, BMl/obesity, and glucose levels) predict risk for T2D?

h. How do other genetic and environmental factors (e.g., race/ethnicity, family history, smoking, diet, exercise level, and other conditions) affect the clinical validity of this test?

4. What are the issues related to the use of TCF7L2 testing in this high-risk population and its impact on patient/consumer outcomes?

a. What are the current management options for high-risk patients/consumers who have a positive TCF7L2 test result in the usual medical model? Would recommendations differ from routine health messages? How might the outcomes change in a direct to consumer model?

b. How could the results of TCF7L2 testing for risk of T2D in this high-risk population impact health behaviors or inform decision making by patients and their health-care providers that affect outcomes?

c. In what ways could the use of TCF7L2 testing in this high-risk population impact clinical outcome (e.g., morbidity/mortality)?

d. What is known about other contextual issues, such as cost-effectiveness, likelihood of behavioral change, and family history considerations?

5. What are the potential harms associated with TCF7L2 testing (e.g., marketing direct to consumers, distress or stigma for a "poor prognosis" result, misinterpretation of results leading to excessive or inadequate treatment, and exploitation of hypervigilant people)?

${ }^{a}$ For example: overweight/obese $\left(\mathrm{BMl} \geq 25 \mathrm{~kg} / \mathrm{m}^{2}\right.$ ) [wt (lbs)/height (in) $\left.\left.{ }^{2}\right] \times 703.0696\right]$ patients with impaired fasting glucose (IFG) or (IGT). IFG is defined as fasting plasma glucose $100 \mathrm{mg} / \mathrm{dl}(5.6 \mathrm{mmol} / \mathrm{l})$ to $125 \mathrm{mg} / \mathrm{dl}(6.9 \mathrm{mmo} / /)$. Impaired glucose tolerance $(I \mathrm{GT})=2$-h plasma glucose $140 \mathrm{mg} / \mathrm{dl}(7.8 \mathrm{mmo} / /)$ to $199 \mathrm{mg} / \mathrm{dl}(11.0 \mathrm{mmo} / \mathrm{l})$ as defined by the American Diabetes Association Standards of Medical Care in Diabetes-2012. ${ }^{19}$

BMI, body mass index; T2D, type 2 diabetes.

were performed through November 2010. The search was rerun in April 2012 to identify recent publications. One investigator (M.M., M.P.D, or S.M.) had primary responsibility of study selection and information extraction for each gene, and results were reviewed by another (usually G.E.P.). Discrepancies were resolved by discussion.

To be included, the study (or meta-analysis) needed to be in English and include information about individuals of
European descent (Caucasian). Articles with only non-European populations were excluded because the tests included in the review were for European descent populations. The primary outcome of T2D was defined in multiple ways: (i) fasting blood glucose level $>126 \mathrm{mg} / \mathrm{dl}$ on two occasions, (ii) hemoglobin A1c test level of $\geq 6.5 \%$, and (iii) oral glucose tolerance test level $>200 \mathrm{mg} / \mathrm{dl}$ after $2 \mathrm{~h} .{ }^{19}$ In clinical scenario 1 (testing in the general population), the presence or absence 
a

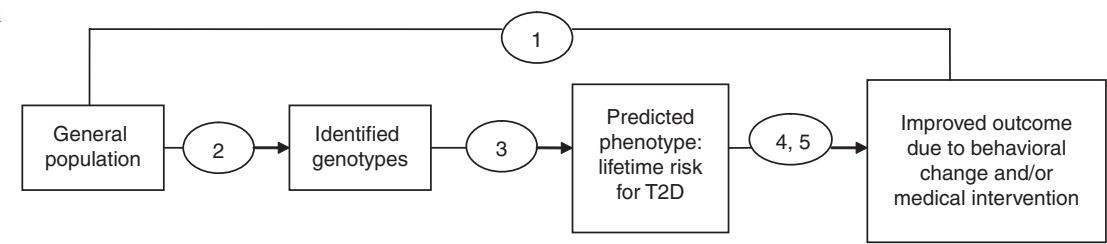

b

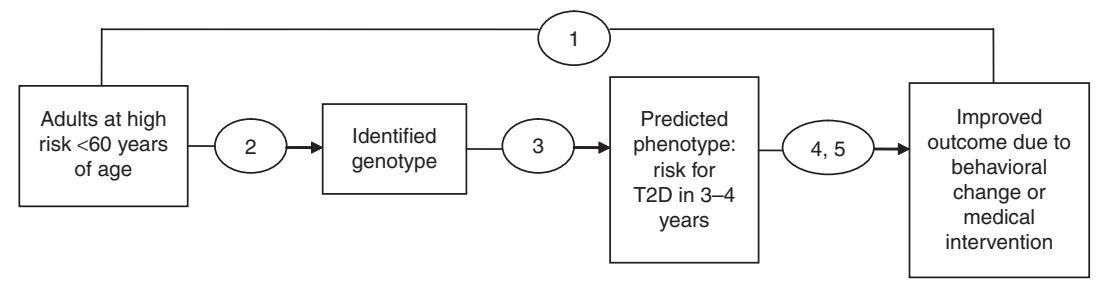

Figure 1 Analytic frameworks. (a) Clinical scenario 1 shows the use of a multigene panel to estimate lifetime risk of developing type 2 diabetes (T2D) and improve patient outcomes. (b) Clinical scenario 2 shows the use of TCF7L2 testing in a high-risk population to determine short-term (3-4 year) risk of developing T2D and improve outcomes. The numbers correspond to the key questions contained in Table 1.

of T2D risk factors is not a consideration. In clinical scenario 2 (testing in high-risk individuals), each person considered for testing must also have traditional risk factor(s) (e.g., obesity, impaired glucose tolerance). For clinical scenario 1, sufficient data needed to be present to express the effect size as an OR with confidence interval (CI). For clinical scenario 2 , sufficient data to quantify the follow-up time period were required.

Summary ORs and corresponding 95\% CIs were derived using a random effects model, either from the original source (published meta-analysis), from a reanalysis of the reported data, or from a new literature summary (Comprehensive MetaAnalysis, Version 2; Englewood, NJ). The preferred summary effect size was an allele-specific OR, with the largest genotypic group used as the referent. When possible, heterogeneity was expressed using the $I^{2}$ statistic $^{20}$ for ease of interpretation and comparison between studies.

The genes included on the genomic panel for clinical scenario 1 were addressed in three tiers. Tier 1 included the four markers found on an early 2010 Google search for "Type 2 Diabetes Risk Tests." That search identified only four gene panels (TCF7L2, CDKAL1, CDKN2A/B, and PPARG). These four markers were included on all T2D panels reviewed; a full analysis was planned for these genes. Tier 2 included four more genes (HHEX, SLC30A8, KCNJ11, and WFS1) that were found to have been added to most panels; these were also subject to complete review. Tier 3 included all remaining gene markers that were identified on only one panel or that were identified as part of an overview of GWA studies. These were subjected to a less stringent analysis that involved calculating a summary OR based only on GWA studies of T2D.

An evaluation guideline to assess the cumulative evidence provided by genetic association studies was published in $2008 .^{21}$ These "Venice criteria" focused on amount of evidence, replication of evidence, and protection from bias. Each criterion is assigned a grade of "A," "B," or "C." The amount of evidence, for example, would receive an " $\mathrm{A}$ " if $>1,000$ cases/controls with the least common genotype were included in computing the effect size estimate, " $B$ " if there were 100-1,000 study subjects, and " $C$ " if $<100$. As suggested in this grading system, ${ }^{21}$ epidemiological evidence for a significant association was rated as "strong" if the meta-analysis received three A grades, "moderate" if it received any $\mathrm{B}$ grade but not any $\mathrm{C}$ grade, and "weak" if it received a $\mathrm{C}$ grade in any of the three criteria. These criteria may not be as relevant for assessing evidence from large GWA studies.

For clinical scenario 1, it was considered unlikely that any data set would be able to adequately assess the independence of multiple gene disease associations. As a way to set a reasonable upper limit, we chose a multiplicative model that assumes each gene marker to be an independent predictor of T2D risk. ${ }^{18}$ Before multiplying a set of ORs, we adjusted them so that there was no overall impact on the population prevalence of T2D risk. ${ }^{22}$

The methods used to estimate the overall clinical validity of genomic panels using multiple markers has been described previously. ${ }^{18}$ Briefly, a Monte Carlo simulation ${ }^{23}$ was used to estimate the distribution of genotypes in individuals with and without T2D using the cumulative allele-specific OR and the risk allele frequency derived from the literature review. Genomic markers were assumed to be independent, and each OR was multiplied to compute the cumulative OR for each individual modeled. Before modeling, the ORs were adjusted such that there was no effect on the prevalence of the disorder. The resulting distributions of cumulative ORs were divided into 20 equal categories (on the logarithmic scale) from 0.1 to 10 .

The common name for each gene included in a commercially available T2D test was searched using the Genopedia option in the HUGE Navigator ${ }^{24}$ for type 2 diabetes to identify meta-analysis of published GWA study findings. If a meta-analysis of GWA studies was not available, the original GWA studies reporting the association for that gene's association with T2D in people of European descent was used. Additional genes not included in commercially available tests were included when results from GWA studies meta-analysis or original GWA studies were available. A table listing all of the 
Table 2 Summary of gene/disease associations for type 2 diabetes in people of European descent by commercially available test ${ }^{\mathrm{a}}$ or select GWA studies

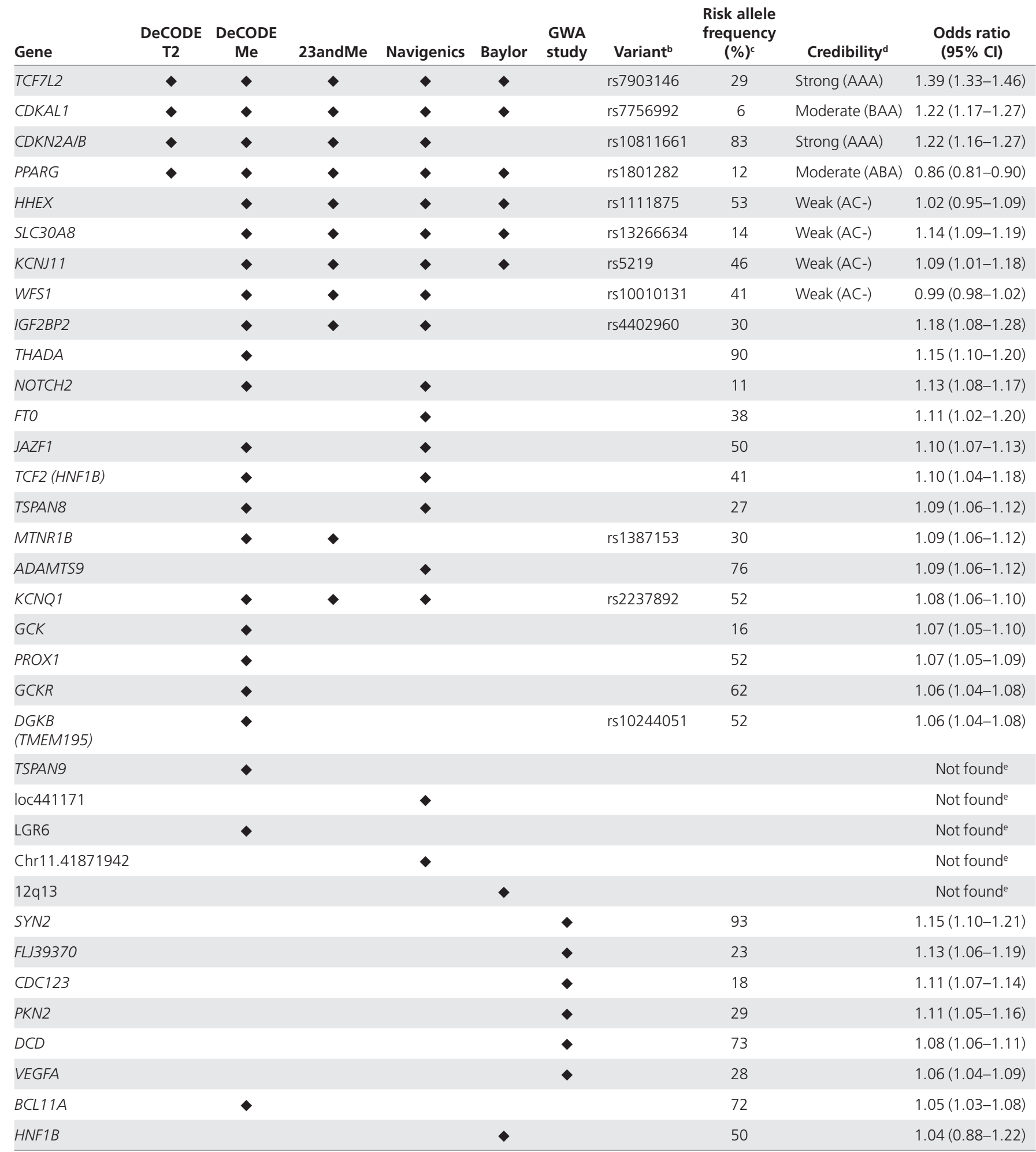

aSNP/loci/gene(s) by company are current as of August 2011. bThe variant(s) are provided by the manufacturer. If no variant was provided by the manufacturer, none is provided in the table. The risk allele frequency is provided if manufacturer provided it or the actual frequency was able to be determined (if the variant was provided). ${ }^{\mathrm{B} B a s e d}$ on criteria ${ }^{21}$ that use three letter grades $(\mathrm{A}, \mathrm{B}$, and $\mathrm{C})$ to determine credibility of a meta-analysis. Amount of evidence: $\mathrm{A}=0 \mathrm{over} 1,000$ cases and controls in least common genotype (assuming 1:1), $B=100-1000, C=<100$. Extent of replication: $A=12<25 \% ; B=1225 \%-50 \% ; C=12>50 \%$ (or single study); $12=(Q-d f) / d f$. Protection from bias: $\mathrm{A}=$ if present, bias might only affect magnitude; $\mathrm{B}=$ no obvious bias, missing information; $\mathrm{C}=$ considerable bias/potential for bias that could impact direction; "- -" not evaluated due to a previous C grade. CI, confidence interval; GWA, genome-wide association; SNP, single-nucleotide polymorphism. ${ }^{\circ}$ Odds ratios were not found for the population studied in searches conducted in HuGE Navigator or PubMed. 
SNPs in commercially available tests and select GWA studies with variants identified as of August 2011 was populated with the risk allele, allele frequency, measure of association (OR), and estimates of variance (95\% CI). Since August 2011, nine additional variants (CENTD2, 1.14 (1.11-1.18); HMGA2, 1.08 (1.04-1.12); IRS1 1.10 (1.08-1.13); KLF14, 1.06 (1.03-1.09); PRC1, 1.06 (1.03-1.08); PROX1, 1.07 (1.05-1.09); TP53INP1, 1.05 (1.03-1.08); ZBED3, 1.07 (1.04-1.10); and ZFAND6 ${ }^{*}, 1.05$ (1.03-1.08)) identified from the Voight and Dupuis GWA studies were added to the deCodeMe test, and two additional previously identified variants (MTNR1B and KCNQ1) were added to the 23andMe test. ${ }^{25,26}$ Overall, the ORs ranged from 1.06 to 1.14 for these nine new variants. The addition of these variants to existing test panels and modeling would not change the final outcomes. Because the aim was more "horizon scanning" than definitively determining the ORs and allele frequency, we chose not to perform an exhaustive review of these later markers.

\section{Clinical utility}

A full systematic review of the entire literature on the clinical utility of any genomic markers/panels was not undertaken. Rather, targeted PubMed searches ${ }^{18}$ were focused on identifying studies reporting on the potential clinical utility only of genetic testing for T2D among the general population and among the defined high-risk population for each clinical scenario. Specific PubMed search strings were "type 2 diabetes" AND "gene intervention OR gene interaction" AND "utility OR outcomes." Studies that reported gene intervention or interaction among the genetic variants that reported clinical outcomes (e.g., increase or decrease of T2D development, increase/ decrease in T2D mortality, change in fasting blood glucose) for T2D included in this report are listed in Supplementary Appendix B online. In general, intermediate outcomes without validation to a clinical outcome (such as reduction in weight) were not included. The exception was the validated clinical outcome of reducing the intermediate measure of plasma glucose levels. ${ }^{27,28}$ Data from the final list of included studies were abstracted into evidence tables including genetic variant, outcome measured, population, and treatment effects stratified by genetic variant and intervention. Articles from the clinical validity review were also reviewed for information related to clinical utility. This review was also updated in April 2012.

\section{Genomic panels for T2D risk}

\section{RESULTS}

The existing genomic panels for T2D risk prediction that are available in the United States were identified as part of a routine horizon scanning process by staff of the Office of Public Health Genomics. ${ }^{29}$ Such tests were available through a health-care provider (Baylor) or through a direct-to-consumer offer (23andMe, DeCodeMe, DeCode T2, and Navigenics). Table 2 lists the five genomic test panels that met these criteria along with the 27 genetic markers included on those panels and eight additional markers found in GWA studies through additional horizon scanning. The deCODE T2 (four markers) and the deCODE
Me (21 markers originally, currently 30) tests are offered by deCODE genetics (Reykjavik, Iceland). 23andMe (9 originally, currently 11; Mountain View, CA), Navigenics (Foster City, CA), and Baylor College of Medicine (Houston, TX) offer interpretations based on 9, 18, and 7 genomic markers, respectively. Only the deCODE T2 and Baylor tests focus solely on T2D. The other tests are part of a larger SNP array for which an analysis of T2D markers is included as part of interpretations for multiple complex disorders (e.g., cardiovascular disease). The top four rows of Table 2 show markers that are included in nearly all panels and have been offered for several years. The next four rows show genes that have been more recently included on most of the identified genomic panels (tier 2). In the next 27 rows are the less commonly included markers used in genomic panels (tier 3); 14 of them are included on only one genomic panel. For completeness, the bottom of the table includes eight additional markers identified through a single summary of three GWA studies of T2D. ${ }^{30-32}$ Several existing panels have increased in size over time, and these are also potential candidates for inclusion.

\section{Analytic validity}

PubMed searches for each of the genes on the genomic panels (e.g., as "TCF7L2" or "TCF7L2 genotyping") and specific terms (e.g., "analytic validity" and "clinical test") identified no articles that provided relevant data on analytic validity. The testing methods used in the research studies reviewed for clinical validity were not considered relevant because they did not include method comparisons and were often not representative of clinical practice. Most importantly, websites from the five companies offering gene/genomic panels related to T2D were reviewed for information related to analytic validity, but no such information was found.

No US laboratories were found in the GeneTest ${ }^{33}$ database offering testing for any of the genes found in the genomic panels in the general population for T2D (clinical scenario 1) or for TCF7L2 and T2D in the high-risk population (clinical scenario 2). In contrast, these panels are part of a whole-exome/wholegenome scan that is interpreted for prediction/risk for several diseases. Two panels (DeCODE T2 and Baylor College of Medicine) ${ }^{34}$ are specifically for T2D risk prediction in the general population. No external proficiency testing program (e.g., American College of Medical Genetics/College of American Pathology) or formal interlaboratory comparison program was found for these panels or for the genes in these panels. ${ }^{35}$ Publications providing relevant information on the analytic validity of testing for these genes were not identified.

\section{Relevant genotyping methodologies}

The literature on allele/SNP genotyping described laboratorydeveloped tests and a large number of commercially available reagents and platforms. ${ }^{36,37}$ In general, genotyping methods have involved discrimination of alleles by primer extension, hybridization, ligation or enzymatic cleavage, and detection using fluorescence, mass, gel electrophoresis, or chemiluminescence. ${ }^{36}$ Mistaken alleles, allelic dropout (i.e., amplification of 
only one of two alleles in a heterozygous individual), and other genotyping errors can result from a number of causes. These include interaction with flanking DNA sequences, low quality/ quantity of DNA in samples, laboratory problems related to reagents/protocols/equipment, and human error (e.g., sample mislabeling or contamination and data entry and interpretation mistakes). Questions remain about causes and rates of genotyping errors in newer technologies (e.g., multiplex assays, chips, and SNP arrays) used in routine clinical practice and their potential impact on patient results. ${ }^{38}$

\section{Clinical validity}

Literature searching. We used HuGE Navigator to search for gene/disease association studies for each gene of interest found in Table 2. The results of literature searches and review can be found in detail for each gene in Supplementary Appendix A online. No new publications were identified from the most recent search.

Clinical scenario 1: association of genomic markers with T2D. Table 2 provides a summary of evidence for the association of individual genes/variants and T2D. The gene abbreviation, genomic panel, and the SNP's identifying RefSNP (rs) number are provided, along with the "risk" allele frequency. It should be noted that the risk allele used in all of these panels is not necessarily causative. Rather, it is most likely a marker, which may help to explain the relatively low ORs obtained. Where determined, the credibility of the evidence (Venice criteria) and associated grades are provided. In the last column, the OR and 95\% $\mathrm{CI}$ are shown. For example, the first row summarizes the information for the TCF7L2 gene SNP rs7903146. The risk allele (T) frequency is $29 \%$ (i.e., $8 \%$ of the population will carry two copies (TT), $42 \%$ one copy (CT), and the remaining $50 \%$ no copies of the risk allele (CC)). The cumulative credibility of evidence is "strong", with three "A" grades for the amount and replication of data and the protection from bias. The consensus per-allele OR is 1.39 (95\% CI: 1.33-1.46). If those individuals with no risk alleles were used as the referent category, then TCF7L2 heterozygous individuals would be assigned an OR of 1.39. Those with two risk alleles would have an OR of $1.93\left(1.39^{\star} 1.39\right)$. In the row summarizing results of the HHEX gene marker, several meta-analyses pointed out the strong association between study sample size and moderate effect size (smaller studies having larger effects), but no attempt was made to grade or account for the potential of publication bias due to the finding of high heterogeneity (replication grade of "C"). A more detailed description of the literature search, how the risk allele frequencies were defined, included studies, tests of heterogeneity, and evaluation of publication bias for each of the combinations listed in Table 2 are all included in Supplementary Appendix B online. Among the eight commonly tested genes evaluated by the Venice criteria, two had strong credibility (TCF7L2 and CDKN2A), two (CDKAL1 and PPARG) were graded as moderate, and four (HHEX, SLC308A, WFS1, and KCNJ11) were graded as having weak credibility. The per-allele OR ranged from 0.86 to 1.39 . In one instance (PPARG), the risk allele frequency is $>50 \%$, indicating that the "risk" allele is actually protective and associated with reduced, rather than increased, risk for T2D.

Among the 19 additional markers included in Table 2 that were also included on a genomic panel, the per-allele ORs ranged from 1.06 to 1.18. Five of these 19 markers had no OR reported, as no published information was found in any search of PubMed or HuGE Navigator. Among the final eight markers identified through GWA studies, the per-allele ORs ranged from 1.04 to 1.15 .

Clinical scenario 1: cumulative effect of multiple genomic markers and T2D. To estimate a reasonable upper limit to the
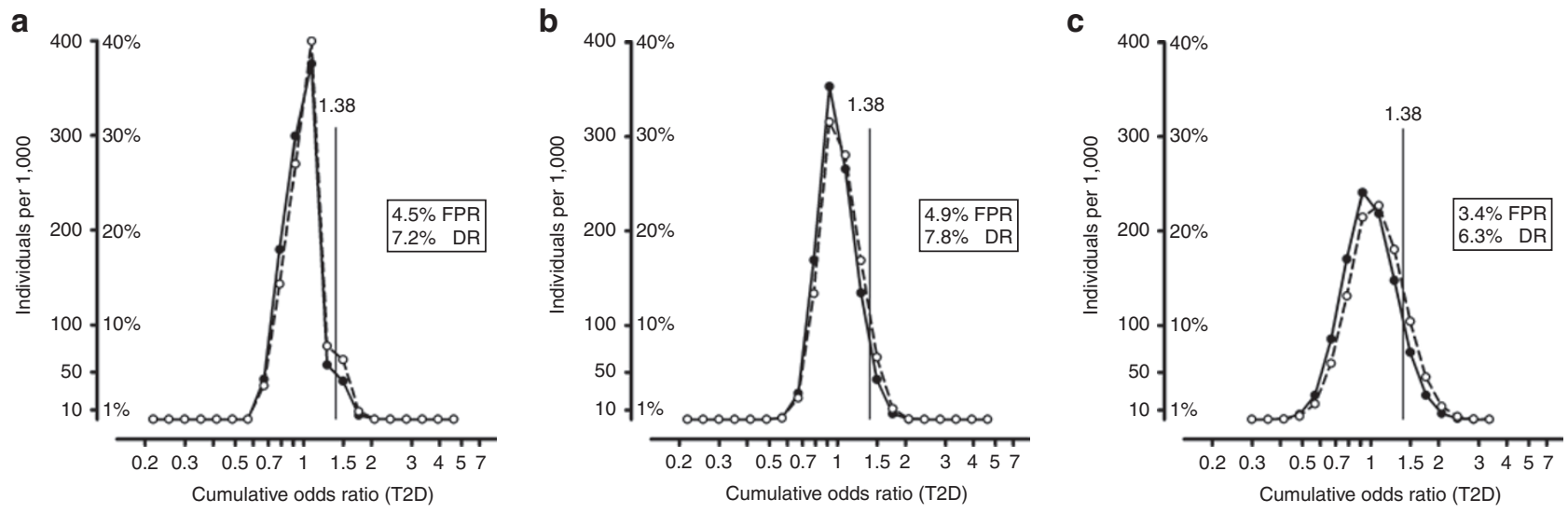

Figure 2 Cumulative odds ratios (ORs) for 4, 8, and $\mathbf{3 0}$ genomic markers to predict type $\mathbf{2}$ diabetes (T2D). (a) The $x$-axis shows the cumulative OR for the four genomic markers with the most credible evidence (TCF7L2, CDKAL1, CDKN2A/B, and PPARG) in a simulated population of individuals developing T2D (dashed line with open circles) and a corresponding population of individuals who do not develop T2D (solid line with filled circles). Details of the model are described in the methods. Each circle represents the number of individuals per 1,000 in each of the 20 intervals (equally spaced on the log scale) from cumulative ORs from 0.1 to 10 . There is considerable overlap with the two groups, indicating poor test performance (false-positive rate: $4.5 \%$ and detection rate: $7.2 \%)$. (b) Includes the same information for the same four genomic markers along with the four next most commonly used markers (HHEX, SLC3OA8, KCNJ11, and WFS1). Performance does not improve appreciably. (c) Includes all 30 genomic markers identified in the evidence review and results in the best, but still poor, performance. DR, detection rate; FPR, false-positive rate. 


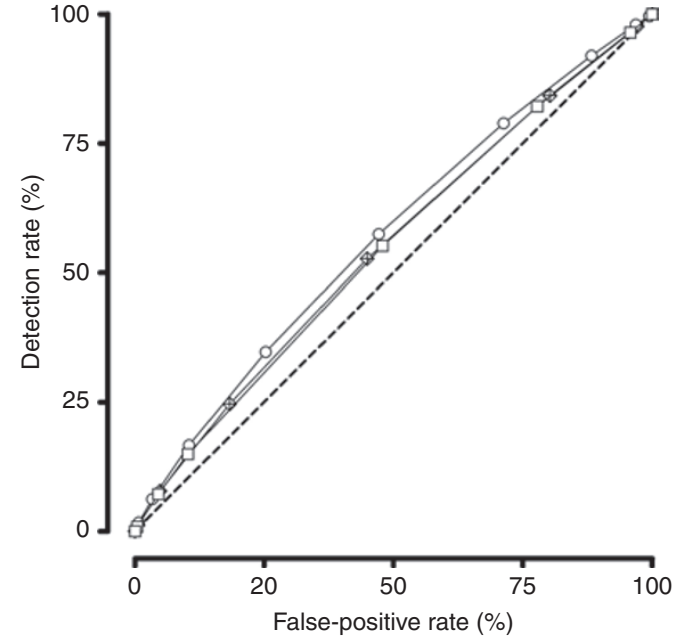

Figure 3 A receiver-operating characteristic curve for three models of type 2 diabetes risk assignment based on genotyping specific SNPs. The detection rate (vertical axis) is plotted versus the false-positive rate (horizontal axis) for 4, 8, and 30 genomic markers associated with type 2 diabetes. The dashed line indicates a "useless" test in which the false-positive rate and detection rate are equal. The four-marker model (open squares) has an area under the curve (AUC) of 0.547 and includes the strongest markers with the highest credibility (Table 2). The eight-marker model (open crossed diamonds) improved AUC to 0.551 , by adding four more widely used markers. The 30-marker model includes all markers on identified panels, along with additional markers identified through the examination of genome-wide association studies. The AUC increases to 0.570 .

effect size that all of these genes might have on T2D risk in a general population, we defined a cumulative OR under the assumption that each genomic marker in Table 2 provides independent information about the risk for $\mathrm{T} 2 \mathrm{D}$ and that none of the effects are synergistic. Three Monte Carlo models were created. The first combined only the four markers, TCF7L2, CDKN2A, $C D K A L 1$, and $P P A R G$, with the highest Venice grades and (ORs $=1.39,1.22,1.22$, and 0.86 , respectively) with highest credible evidence (Venice grades $=$ strong or moderate). Figure 2a shows the overlapping distributions for the simulated cumulative OR for individuals with (open circles, dashed line) and without (filled circles, solid line) T2D. Using a cumulative OR of 1.38 as a cutoff (the demarcation between the 12th and 13th of the 20 intervals), $7.2 \%$ of T2D and $4.5 \%$ of normal individuals received cumulative ORs at or above this level. This interval was chosen under the assumption that any screening test identifying a "high-risk" population would likely have a relatively low falsepositive rate of approximately 5-10\%. A second model used the first eight makers (Table 2) for which a structured review was performed (Figure $\mathbf{2 b}$ ). Even though four more markers were added, the impact on performance is small, with a detection and false-positive rate of $7.8 \%$ and $4.9 \%$, respectively (using the same $1.38 \mathrm{OR}$ as a cutoff). Finally, a third model used all 30 markers (Table 2) that had an OR identified (Figure 2c). The effect of adding 23 more markers is to spread out the two distributions, but little improvement in performance is seen (detection and false-positive rates of $6.3 \%$ and $3.4 \%$, respectively). Figure 3 shows the relevant receiver-operating characteristic curves for all three models. The curves are similar, with areas under the curve of $0.547,0.551$, and 0.570 , respectively. A recently published simulation study ${ }^{39}$ concluded that improvements in net reclassification without an increase in the AUC are unlikely to result in a clinically useful improvement in performance. Even though more associations between genomic markers and T2D are reported almost monthly, the ORs are always low, and their addition to an existing panel will be minimal. In addition, the assumption of independence of markers would be less plausible, when the number of markers grows too large.

Clinical scenario 2: association of TCF7L2 with T2D in highrisk populations. No published meta-analyses were identified that systematically reviewed the literature regarding genotyping individuals at high risk of T2D for TCF7L2, and using the results to predict the probability of developing T2D within 4 or 5 years. No recent studies were identified as part of our update. For the rs7903146 SNP, two studies (in white, northern European populations) were included for the TT versus CC comparison. ${ }^{3,40}$ Summary measures were calculated using individual studies of TCF7L2 in high-risk populations. The overall OR was 1.66 (95\% CI: $1.22-2.27 ; P=0.001)$ for TT versus CC. For CT versus CC, the OR was 1.12 (95\% CI: $0.85-1.48 ; P=0.4$ ). There was no evidence of heterogeneity $\left(I^{2}=0 \%\right)$ for the former, and moderate heterogeneity for the latter $\left(I^{2}=25 \%\right)$. However, evidence for heterogeneity is limited because only two studies were pooled. Neither of the studies reported on $>500$ cases.

Four studies examined the predicted risk of developing T2D using TCF7L2 in combination with other markers, ${ }^{41-44}$ many of which were included in the review of clinical scenario 1 . Because of the expanded panel, they are not directly relevant, but review of these results might provide indirect evidence for clinical validity in this setting. Each of the studies was in a similar high-risk population setting, and all used nongenetic factors in estimating risk. The net reclassification index (NRI) ${ }^{45}$ was used to determine the improvement of risk classification gained by including the results of genomic panel testing in addition to traditional risk factors. The NRI is most informative if the reclassification is first reported separately for both the cases (a positive percentage indicates improved risk classification among individuals who developed T2D) and the controls (a negative percentage indicates improved risk classification among individuals who did not develop T2D). The difference of these (NRI $\left.{ }_{\text {case }}-\mathrm{NRI}_{\text {control }}\right)$ is the overall NRI. Overall, the data from these four studies provide moderate evidence of little or no improvement in reclassification of risk, even if TCF7L2 were the main reason for any improvement:

- In 2008, Meigs et al. ${ }^{43}$ reported a prospective cohort study of 2,377 adults in which 255 developed T2D within 28 years. A panel of 20 SNPs (including TCF7L2) was used to retrospectively genotype stored samples. The case, control, and total NRI were $1.2 \%,-1.1 \%$, and $2.1 \%$, respectively $(P$ $=0.17$ ).

- In 2010, Talmud et al..$^{42}$ reported a prospective cohort study of 5,535 adults in which 302 developed T2D within 5 years. 
A panel of eight SNPs (including TCF7L2) was run on stored samples. The case, control, and total NRI were $6.4 \%$, $2.6 \%$, and $3.8 \%$, respectively $(P=0.24)$.

- In 2008, Lyssenko et al. $^{41}$ reported on two prospective cohorts: 16,000 from Sweden and 3,000 from Finland. Over the next 23 years, 2,201 developed T2D. A panel of 11 SNPs (including TCF7L2) was tested on stored sera. The case, control, and total NRI were $2.6 \%,-1.9 \%$, and $4.5 \%$, respectively $(P<0.001)$.

- In 2010, De Miguel-Yanes et al..$^{44}$ updated the information regarding the cohort published in $2008 .{ }^{43}$ There were now 446 individuals with T2D, and the panel was increased to 40 SNPs. Only the total NRI of $1.9 \%$ was reported $(P=0.2)$.

\section{Clinical utility}

A total of 79 studies were identified using our search strategy to evaluate clinical utility. After reviewing abstracts and full-text publications, three studies met inclusion criteria. Two randomized controlled trials $s^{3,9}$ and one prospective cohort ${ }^{8}$ reported intermediate and clinical outcomes for gene-intervention interaction ranging between 10 weeks and 7 years of follow-up. Patient populations included both those at high risk (impaired glucose tolerance and/or body mass index $>30$ ) as well as individuals from the general population. Data from these three studies were abstracted into evidence tables including genetic variant, population studied, outcome measured, and treatment effects stratified by genetic variant and intervention (Table 3 ).

Clinical scenario 1: cumulative effect of multiple genomic markers and T2D. The EPIC-Potsdam study ${ }^{8}$ was a prospective case-cohort design with 773 individuals with T2D and 2,225 randomly selected population-based controls followed for an average of 7.1 years (Table 3). The risk of developing T2D was associated with the TCF7L2 T allele (hazard ratio: 1.51) and was compared with daily consumption of whole grains stratified by TCF7L2 genotype. This observational study found a protective effect of whole-grain intake on diabetes risk exclusively applied to the CC genotype (hazard ratio relative to overall effect of 0.86 per $50 \mathrm{~g} /$ day), whereas those carrying at least one $\mathrm{T}$ allele showed no such benefit (hazard ratio relative to overall effect of 1.08 per $50 \mathrm{~g} /$ day).

Clinical scenario 2: association of TCF7L2 with T2D in highrisk populations. The Diabetes Prevention Program ${ }^{3}$ was a multicenter randomized controlled trial comparing the effects of metformin and intensive lifestyle intervention aimed at reducing the incidence of $\mathrm{T} 2 \mathrm{D}$ among obese individuals who already had impaired glucose tolerance testing results. Treatment with metformin was initiated at $850 \mathrm{mg}$ administered orally once daily and raised to twice daily after the first month. The goal of the lifestyle intervention was to reduce body weight by $7 \%$ through a low-fat, low-carbohydrate diet with an average of 150 min of exercise per week. TCF7L2 genotyping was retrospectively performed on 3,549 patients (average age: 53.7 years and average body mass index: $34 \mathrm{~kg} / \mathrm{m}^{2}$ ). The expectation was that in the placebo arm, the TT (at risk) population would have a higher incidence of T2D. At the end of an average of 3 years of follow-up, there was a $24 \%$ reduction in incident T2D in the metformin treatment arm and a 53\% reduction in the lifestyle intervention arm as compared with placebo (hazard ratio: 1.81) in the at-risk (TT) population (row 2, Table 3). None of the interactions of genotype and intervention were significantly different from the placebo group.

The NUGENOB study ${ }^{9}$ was a multicenter randomized openlabel trial that compared the benefits of two dietary interventions $^{8}$ with intermediate outcomes relevant to T2D. Only the surrogate outcome of plasma fasting glucose is relevant to this review. Subjects ranged between the ages of 20 and 50 years with a body mass index of $30 \mathrm{~kg} / \mathrm{m}^{2}$ or greater. A low-fat diet (20-25\% of energy from fat) was compared with a high-fat diet ( $40-45 \%$ from fat). The change in fasting plasma glucose was measured at baseline and at the end of a 10-week intervention period. The impact of the high- and low-fat diets was not associated with TCF7L2 genotype (row 3, Table 3).

Table 3 Clinical utility from genotype-intervention interaction to prevent T2D in high-risk populations

\begin{tabular}{|c|c|c|c|c|c|c|c|c|c|}
\hline \multirow[b]{3}{*}{ Study } & \multirow[b]{3}{*}{ Population } & \multirow[b]{3}{*}{ Outcome } & \multirow[b]{3}{*}{ Gene (SNP) } & \multicolumn{2}{|c|}{ Genotype } & \multicolumn{4}{|c|}{ Intervention effect size $(95 \% \mathrm{Cl})$} \\
\hline & & & & \multirow{2}{*}{\multicolumn{2}{|c|}{ ( $\mathrm{T}$ is risk allele) }} & \multirow[b]{2}{*}{ None } & \multicolumn{3}{|c|}{ Whole-grain consumption } \\
\hline & & & & & & & \multicolumn{2}{|c|}{ CC } & $\mathrm{CT}+\mathrm{TT}$ \\
\hline & \multirow{2}{*}{$\begin{array}{l}\text { Adults in } \\
\text { general }\end{array}$} & & & & & \multicolumn{4}{|c|}{ Intervention effect size $(95 \% \mathrm{Cl})$} \\
\hline & & & & & & Placebo & Metf & $\min$ & Lifestyle \\
\hline & \multirow{3}{*}{$\begin{array}{l}\text { Overweight } \\
\text { with IGT }\end{array}$} & \multirow{3}{*}{$\begin{array}{l}\text { Incident T2D } \\
\text { within } 4 \text { years }\end{array}$} & \multirow{3}{*}{$\begin{array}{l}\text { TCF7L2 } \\
\text { (rs7903146) }\end{array}$} & \multicolumn{6}{|c|}{ Intervention effect size (SD) stratified by TCF7L2 genotype } \\
\hline & & & & \multicolumn{2}{|c|}{$\mathrm{CC}$} & \multicolumn{2}{|c|}{ CT } & \multicolumn{2}{|r|}{$\mathrm{TT}$} \\
\hline & & & & Low fat & High & Low fat & High fat & Low fat & High fat \\
\hline Grau et al. ${ }^{9}$ & $\begin{array}{l}\text { Obese } \\
\text { adults }\end{array}$ & $\begin{array}{l}\text { Change in } \\
\text { fasting glucose }\end{array}$ & $\begin{array}{l}\text { TCF7L2 } \\
\text { (rs7903146) }\end{array}$ & $-0.1(0.5)^{\mathrm{a}}$ & -0.1 & $-0.1(0.5)$ & $-0.2(0.5)$ & $-0.2(0.3)$ & $0.01(0.7)$ \\
\hline
\end{tabular}

$\mathrm{Cl}$, confidence interval; IGT, impaired glucose tolerance; SNP, single-nucleotide polymorphism; T2D, type 2 diabetes.

aMean preintervention fasting glucose minus postintervention fasting glucose in millimoles per liter, (SD). 
Recent studies have shown the effectiveness of bariatric surgery in reducing or reversing the negative effects of T2D. In a recent meta-analysis of 35 studies reporting laparoscopic adjustable gastric banding, ${ }^{46}$ weight loss over the first 2 years averaged $47 \%$, whereas remission or improvement in diabetes varied from $53 \%$ to $70 \%$. However, the studies were generally of low quality. Only one study was identified that evaluated TCF7L2 genotype among individuals undergoing bariatric surgery. ${ }^{47}$ Although these studies were performed in patients with T2D, the procedure would likely reduce the risk of developing T2D. They performed mRNA expression analysis on mRNA isolated from liver and found that TCF7L2 expression was unrelated to age or glycated hemoglobin but was associated with body mass index, depending on whether the obese patient had an existing diagnosis of T2D. It should be noted that TCF7L2 has a complex transcription and splicing pattern that varies between tissues, so analyses that do not distinguish between transcripts may obscure important differences.

Update on the availability and content of genomic panels for T2D. This targeted review addresses only those genes included on genomic profiles aimed at T2D that were available when the study was undertaken in mid-2010. Because it is possible that additional or updated panels may now exist, we repeated our search strategy in August 2011 and April 2012. Two of the genomic panels in Table 2 have updated their panels. DecodeMe has added nine variants to its panel, and 23 andMe has added two variants to its panel. Two new GWA studies were identified with new associated SNPs, ${ }^{25,26}$ none of these were associated with ORs $>1.15$. No new genomic panels for $\mathrm{T} 2 \mathrm{D}$ risk were identified.

\section{Quality of evidence}

\section{DISCUSSION}

Analytic validity. The quality of evidence for analytic validity is inadequate (scale: inadequate, adequate, and convincing). For the panels/test described in Table 2, no publications were identified that provided relevant information on the analytic validity of testing for these gene variants. For many of the panels, the testing platform was not specified.

Clinical validity. The setting for clinical scenario 1 (Figure 1) was the general population, with testing of genetic variants to identify individuals at risk for T2D. The quality of evidence for clinical validity for clinical scenario 1 varied widely among the 27 genes/variants identified on one or more of the T2D panels. The Venice grades ranged from strong to weak among the eight variants commonly found (Table 2). The strongest evidence was for the rs7903146 SNP in the TCF7L2 gene, with a per-allele OR of 1.39. We identified no information for four of the variants. Horizon scanning for relevant GWA studies results identified an additional eight potentially useful variants, but the per-allele ORs were all 1.15 or smaller. In an attempt to quantify the potential for this type of testing to provide useful information, we created a model system under the assumption that the per-allele ORs for each of the reported variants provide independent information regarding the risk of developing T2D. Using a combination of the four most widely offered variants, the estimated AUC was 0.547. By adding an additional four, or including all 27 quantified variants to the model, the AUC improved slightly to 0.551 and 0.570 , respectively. Even if these test panel results are independent of traditional risk factors for $\mathrm{T} 2 \mathrm{D}$, they are in the range of AUCs considered unlikely to provide important clinically relevant improvements to T2D risk classification in the general population. ${ }^{48}$ Our modeling might have overestimated the combined effect if some markers are related (e.g., in the same biological pathway). Alternatively, if some markers interact to provide effect sizes that are larger than the product of the two, our model would underestimate the effect size. Therefore, the results of modeling the effect size for T2D or other genomic panels should only be considered an estimate.

The setting for clinical scenario 2 was a high-risk population (defined by obesity, impaired glucose tolerance, or both), with testing of a single genetic variant (the rs7903146 SNP in the TCF7L2 gene) to identify those individuals who will develop T2D. Two studies reported directly relevant data, with overall OR of 1.66 for the comparison of TT versus CC and 1.12 for the CT versus CC comparison. These limited data are consistent with the 1.38 per-allele OR for this variant in the general population. Four other studies used the same TCF7L2 variant in a genomic panel with other T2Drelated variants to explore the reclassification of high-risk individuals and found little, if any, improvement. Overall, there is moderate evidence of, at most, minor improvement in risk classification using TCF7L2 genotyping in a population already at high risk of T2D.

Clinical utility. Two studies provided limited data on genotyperelated differences in interventions aimed at reducing the risk of developing T2D in a general adult population (Table 3). Both restricted genotyping to TCF7L2 and both confirmed the higher risk associated with the high-risk homozygous genotype (Table 3). One of the studies examined the dietary intake of whole grains and found that individuals with at least one $\mathrm{T}$ allele did not benefit, whereas those without a $\mathrm{T}$ allele showed some reduction in risk, but the difference was not significant. Another report focused on the use of metformin and on lifestyle changes. None were found to interact with the TCF7L2 genotype. One study addressed a dietary intervention in the high-risk population and found no significant association with genotype and the change in fasting glucose. Overall, the quality of evidence for clinical utility in both scenarios is considered insufficient due to limited studies, limited interventions, and small sample sizes.

\section{GAPS IN KNOWLEDGE}

- Little or no available information on the analytic validity of T2D panels, either in the published literature, or on the company websites. Often, it was not possible to even determine the testing platform or methodology being used. 
- Which of the gene/variant associations identified might benefit from further localization of the causative variant to improve their credibility and effect size.

- Which, if any, of the gene/disease associations identified with moderate or weak credibility might be overestimated due to potential biases (e.g., publication bias).

- How multiple genomic markers for T2D should be combined and the types of data needed to inform these models.

- How genetic background/ethnicity would influence the OR for any particular variant.

- Alternative strategies for the prevention of T2D and how genomic markers might impact these strategies.

- Clinical trials to inform individuals with specific genotypes (e.g., TCF7L2) and how behavioral (i.e., diet and exercise) and/or pharmacological (i.e., metformin) changes effective T2D risk in the general or high-risk populations.

- How genomic markers for T2D can be incorporated into risk algorithms that include family history and whether genomic markers provide useful risk predication when family history is lacking (i.e., adoption cases).

\section{RESEARCH AGENDA}

Laboratories performing analytic validation studies for T2D or other genomic panels should consider publishing their detailed results in peer-reviewed journals, as a way to build the evidence base for reliable testing. A consensus method should be developed to handle data with poor credibility and/or the existence of possible bias that could have a nontrivial impact on the effect size. This would allow more consistent and reliable modeling to occur. Further work on standardizing genotype models, summarizing/evaluating the literature, combinations of genomic markers, and combinations of genomic and nongenomic markers should be continued. ${ }^{21,49}$

\section{SUPPLEMENTARY MATERIAL}

Supplementary material is linked to the online version of the paper at http://www.nature.com/gim

\section{ACKNOWLEDGMENTS}

Funding for this report was provided by the Office of Public Health Genomics, Centers for Disease Control and Prevention, through a contract (200-2003-01396-0128) with McKing Consulting Corporation. We thank the Genomic Applications in Practice and Prevention Working Group members of the Technical Evaluation Panel, Margaret Piper, PhD, MPH (Blue Cross/Blue Shield Association), Doug Campos-Outcalt, MD, MPA (University of Arizona College of Medicine), Theodore Ganiats, MD (University of California, San Diego), and Nancy L. Fisher, RN, MD, MPH (Washington State Health Care Authority) who provided guidance and comments on drafts of this article. We also thank the reviewers of the manuscript, including Jose Florez (Massachusetts General Hospital), Catherine McKeon (NIH-NIDDK), and Laura Scott (University of Michigan).

\section{DISCLOSURE}

The authors declare no conflict of interest.

\section{REFERENCES}

1. Centers for Disease Control and Prevention. National Diabetes Fact Sheet: National Estimates and General Information on Diabetes and Prediabetes in the United States 2011. CDC: Atlanta, GA, 2011.

2. Knowler WC, Barrett-Connor E, Fowler SE, et al.; Diabetes Prevention Program Research Group. Reduction in the incidence of type 2 diabetes with lifestyle intervention or metformin. N Engl J Med 2002;346:393-403.

3. Florez JC, Jablonski KA, Bayley N, et al.; Diabetes Prevention Program Research Group. TCF7L2 polymorphisms and progression to diabetes in the Diabetes Prevention Program. N Engl J Med 2006;355:241-250.

4. Moore AF, Jablonski KA, McAteer JB, et al.; Diabetes Prevention Program Research Group. Extension of type 2 diabetes genome-wide association scan results in the diabetes prevention program. Diabetes 2008;57:2503-2510.

5. Florez JC, Jablonski KA, Sun MW, et al.; Diabetes Prevention Program Research Group. Effects of the type 2 diabetes-associated PPARG P12A polymorphism on progression to diabetes and response to troglitazone. J Clin Endocrinol Metab 2007:92:1502-1509.

6. Florez JC, Jablonski KA, Kahn SE, et al. Type 2 diabetes-associated missense polymorphisms KCNJ11 E23K and ABCC8 A1369S influence progression to diabetes and response to interventions in the Diabetes Prevention Program. Diabetes 2007;56:531-536.

7. Jablonski KA, McAteer JB, de Bakker PI, et al.; Diabetes Prevention Program Research Group. Common variants in 40 genes assessed for diabetes incidence and response to metformin and lifestyle intervention in the diabetes prevention program. Diabetes 2010;59:2672-2681.

8. Fisher E, Boeing H, Fritsche A, Doering F, Joost HG, Schulze MB. Whole-grain consumption and transcription factor-7-like 2 (TCF7L2) rs7903146: gene-diet interaction in modulating type 2 diabetes risk. Br J Nutr 2009;101:478-481.

9. Grau K, Cauchi S, Holst C, et al. TCF7L2 rs7903146-macronutrient interaction in obese individuals' responses to a 10-wk randomized hypoenergetic diet. Am J Clin Nutr 2010;91:472-479.

10. Valdez R. Detecting undiagnosed type 2 diabetes: family history as a risk factor and screening tool. J Diabetes Sci Technol 2009;3:722-726.

11. Harrison TA, Hindorff LA, Kim H, et al. Family history of diabetes as a potential public health tool. Am J Prev Med 2003;24:152-159.

12. Kobberling J, Tillil H. Empirical risk figures for first degree relatives of non-insulin dependent diabetics. In: Kobberling J, Tillil H eds. The Genetics of Diabetes Mellitus. Academic Press: London, 1982:201-209.

13. Teutsch SM, Bradley LA, Palomaki GE, et al.; EGAPP Working Group. The Evaluation of Genomic Applications in Practice and Prevention (EGAPP) Initiative: methods of the EGAPP Working Group. Genet Med 2009;11: 3-14.

14. Haddow JE, Palomaki GE. ACCE: a model process for evaluating data on emerging genetic tests. In: Khoury M, Little J, Burke W (eds). Human Genome Epidemiology: A Scientific Foundation for Using Genetic Information to Improve Health and Prevent Disease. Oxford University Press: Oxford, 2003:217-233.

15. Ge D, Dong Y, Wang X, Treiber FA, Snieder H. The Georgia Cardiovascular Twin Study: influence of genetic predisposition and chronic stress on risk for cardiovascular disease and type 2 diabetes. Twin Res Hum Genet 2006;9: 965-970.

16. Chambers EC, Heshka S, Gallagher D, Wang J, Pi-Sunyer FX, Pierson RN Jr. Serum magnesium and type-2 diabetes in African Americans and Hispanics: a New York cohort. J Am Coll Nutr 2006;25:509-513.

17. Yu W, Wulf A, Yesupriya A, Clyne M, Khoury MJ, Gwinn M. HuGE Watch: tracking trends and patterns of published studies of genetic association and human genome epidemiology in near-real time. Eur J Hum Genet 2008;16:1155-1158.

18. Palomaki GE, Melillo S, Neveux L, et al. Use of genomic profiling to assess risk for cardiovascular disease and identify individualized prevention strategies-a targeted evidence-based review. Genet Med 2010;12:772-784.

19. American Diabetes Association. Standards of medical care in diabetes-2012. Diabetes Care 2012;35(suppl 1):S11-S63.

20. Higgins JP, Thompson SG, Deeks JJ, Altman DG. Measuring inconsistency in meta-analyses. BMJ 2003;327:557-560.

21. loannidis JP, Boffetta P, Little J, et al. Assessment of cumulative evidence on genetic associations: interim guidelines. Int J Epidemio/ 2008;37:120-132.

22. Janssens $A C$, van Duijn CM. Five genetic variants associated with prostate cancer. N Engl J Med 2008;358:2739; author reply 2741.

23. Fan X, Felsovalyi A, Sivo S, Keenan S. SAS for Monte Carlo Studies: A Guide for Quantitative Researchers. SAS Publishing, 2002, http://support.sas.com/ publishing/. 
24. Yu W, Gwinn M, Clyne M, Yesupriya A, Khoury MJ. A navigator for human genome epidemiology. Nat Genet 2008;40:124-125.

25. Voight BF, Scott LJ, Steinthorsdottir V, et al.; MAGIC investigators; GIANT Consortium. Twelve type 2 diabetes susceptibility loci identified through largescale association analysis. Nat Genet 2010;42:579-589.

26. Dupuis J, Langenberg C, Prokopenko I, et al.; DIAGRAM Consortium; GIANT Consortium; Global BPgen Consortium; Anders Hamsten on behalf of Procardis Consortium; MAGIC investigators. New genetic loci implicated in fasting glucose homeostasis and their impact on type 2 diabetes risk. Nat Genet 2010;42:105-116.

27. Fonseca $V A$. Identification and treatment of prediabetes to prevent progression to type 2 diabetes. Clin Cornerstone 2007;8:10-18; discussion 19.

28. Fonseca VA. Identification and treatment of prediabetes to prevent progression to type 2 diabetes. Clin Cornerstone 2008;9:51-59; discussion 60.

29. Gwinn M, Grossniklaus DA, Yu W, et al. Horizon scanning for new genomic tests. Genet Med 2011;13:161-165.

30. Saxena R, Voight BF, Lyssenko V, et al. Genome-wide association analysis identifies loci for type 2 diabetes and triglyceride levels. Science 2007:316:1331-1336.

31. Waters KM, Stram DO, Hassanein MT, et al. Consistent association of type 2 diabetes risk variants found in Europeans in diverse racial and ethnic groups. PLoS Genet 2010;6:e.1001078.

32. Zeggini E, Scott L, Saxena R, et al.; Wellcome Trust Case Control Consortium. Meta-analysis of genome-wide association data and large-scale replication identifies additional susceptibility loci for type 2 diabetes. Nat Genet 2008;40:638-645.

33. Wang H, Dou KF, Yang XJ, et al. [A case report of simultaneous liver, pancreasduodenum, and kidney transplantation in a patient with post-hepatitic cirrhosis combined with uremia and insulin-dependent diabetes related to chronic pancreatitis]. Zhonghua Yi Xue Za Zhi 2006;86:2421-2424.

34. Personalized Medical Genomic Profile (PMGP) https://www.bcm.edu/ geneticlabs/test_detail.cfm?testcode $=7000$ (2012).

35. Hung YJ, Kuo SW, Wang CH, Chang HY, Hsieh SH, Landen H. Postmarketing surveillance of acarbose treatment in Taiwanese patients with type 2 diabetes mellitus. Clin Drug Investig 2006;26:559-565.

36. Kim S, Misra A. SNP genotyping: technologies and biomedical applications. Annu Rev Biomed Eng 2007;9:289-320.
37. Ragoussis J. Genotyping technologies for genetic research. Annu Rev Genomics Hum Genet 2009;10:117-133.

38. Pompanon F, Bonin A, Bellemain E, Taberlet P. Genotyping errors: causes, consequences and solutions. Nat Rev Genet 2005;6:847-859.

39. Mihaescu R, van Hoek M, Sijbrands EJ, et al. Evaluation of risk prediction updates from commercial genome-wide scans. Genet Med 2009;11:588-594.

40. Wang J, Kuusisto J, Vänttinen $\mathrm{M}$, et al. Variants of transcription factor 7 -like 2 (TCF7L2) gene predict conversion to type 2 diabetes in the Finnish Diabetes Prevention Study and are associated with impaired glucose regulation and impaired insulin secretion. Diabetologia 2007;50:1192-1200.

41. Lyssenko V, Jonsson A, Almgren P, et al. Clinical risk factors, DNA variants, and the development of type 2 diabetes. N Eng/ J Med 2008;359:2220-2232.

42. Talmud PJ, Hingorani AD, Cooper JA, et al. Utility of genetic and non-genetic risk factors in prediction of type 2 diabetes: Whitehall II prospective cohort study. BMJ 2010;340:b4838.

43. Meigs JB, Shrader P, Sullivan LM, et al. Genotype score in addition to common risk factors for prediction of type 2 diabetes. N Engl J Med 2008;359:22082219

44. de Miguel-Yanes JM, Shrader P, Pencina MJ, et al.; MAGIC Investigators; DIAGRAM+ Investigators. Genetic risk reclassification for type 2 diabetes by age below or above 50 years using 40 type 2 diabetes risk single nucleotide polymorphisms. Diabetes Care 2011;34:121-125.

45. Pencina MJ, D'Agostino RB Sr, D'Agostino RB Jr, Vasan RS. Evaluating the added predictive ability of a new marker: from area under the ROC curve to reclassification and beyond. Stat Med 2008;27:157-172; discussion 207.

46. Dixon JB, Murphy DK, Segel JE, Finkelstein EA. Impact of laparoscopic adjustable gastric banding on type 2 diabetes. Obes Rev 2012;13:57-67.

47. Hindle AK, Brody F, Tevar R, et al. TCF7L2 expression in diabetic patients undergoing bariatric surgery. Surg Endosc 2009;23:700-704.

48. Mihaescu R, van Zitteren $M$, van Hoek M, et al. Improvement of risk prediction by genomic profiling: reclassification measures versus the area under the receiver operating characteristic curve. Am J Epidemio/ 2010;172:353-361.

49. Hlatky MA, Greenland P, Arnett DK, et al.; American Heart Association Expert Panel on Subclinical Atherosclerotic Diseases and Emerging Risk Factors and the Stroke Council. Criteria for evaluation of novel markers of cardiovascular risk: a scientific statement from the American Heart Association. Circulation 2009:119:2408-2416. 